\title{
Assessment of mandarin and peach canopy water status using visible multispectral imagery
}

\author{
János Kriston-Vizi a, ${ }^{\text {*,1 }}$ Mikio Umeda ${ }^{a}$ Kumi Miyamotob \\ ${ }^{a}$ Laboratory of Field Robotics and Precision Agriculture, Graduate School of \\ Agriculture, Kyoto University, Sakyo-ku, Kyoto, 606-8502, Japan \\ ${ }^{\mathrm{b}}$ Fruit Tree Experiment Station, Wakayama Research Center of Agriculture, \\ Forestry and Fisheries, 643-0022, Japan
}

\begin{abstract}
Producing high quality mandarin or peach fruits depends on the ability to maintain an optimal level of water stress in the plant during the sugar accumulation period. This study investigates the use of visible imaging for monitoring water stress. Experiments were conducted on satsuma mandarin (Citrus unshiu Marc. var. Satsuma) and peach (Persica vulgaris Mill.) in southwestern Japan. Water stress was induced using plastic mulching at field-scale. Images of crop leaves as well as orchard canopy were taken in near infrared $(760-900 \mathrm{~nm})$, red $(580-680 \mathrm{~nm})$ and green $(490-580 \mathrm{~nm})$ spectral channels on six days. Neutral Gray Card was used as reflectance standard. Monitored crop parameter was leaf water potential. A reflectance difference was found between water stressed and non water stressed mandarin and peach plants in red and green spectral channels both at individual leaf level and field-scale canopy level. A moderate correlation was found between red reflectance and leaf water potential as well as between green reflectance and leaf water potential. Future research should aim at developing visible imaging into a precision irrigation tool applicable to mandarin and peach crops.
\end{abstract}

Key words: image analysis, citrus, satsuma, peach, multispectral imagery, remote sensing, leaf water potential, field spectroscopy

* Corresponding author.

Email address: janos@kristonvizi.hu (János Kriston-Vizi).

1 Supported by Japanese Society for the Promotion of Science (JSPS). 


\section{Introduction}

Water is a major factor effecting both quality and quantity of satsuma mandarin (Citrus unshiu Marc. var. Satsuma) and peach (Persica vulgaris Mill.). While excessive water leads to increased yield and vegetative growth, important fruit quality parameters such as sugar content, acidity, and pigment development are also effected negatively. On the other hand, severe water stress causes reduced shoot growth and assimilative activity, and inhibits fruit sugar accumulation and wood maturation. A moderate level water stress applied in the proper vegetative period can be beneficial in mandarin and peach cultivation as it enhances fruit quality parameters without significantly compromising yield. In order to achieve optimal water stress, tree water status must be measured accurately and reliably with the aim of maintaining a predetermined level of slight stress. A crop water status can be estimated directly either by the sensing of plant water stress parameters, like leaf and stem water potential, leaf conductance, or indirectly by using indicators of water status based on the analysis of plant growth or physiological responses known to be indicative of water deficits (Jones, 2004). For plant physiology studies, Slatyer and Taylor (1960) introduced the term 'water potential' $(\psi)$, and later defined it in terms of chemical potential of water $(\mu)$ divided by the partial molal volume of water

$$
\psi=\left(\mu-\mu_{0}\right) / V_{w}=-\left(R T / V_{w}\right) \log _{e}\left(e / e_{s}\right)
$$

where $V_{w}$ is the partial molal volume of water, $\mu_{0}$ is the chemical potential of pure water at a reference level, $\mathrm{R}$ is the universal gas constant, $\mathrm{T}$ is the temperature $(\mathrm{K})$, and $e / e_{s}$ is the vapour pressure in equilibrium with the water containing matrix divided by the saturation vapour pressure at that temperature. The total water potential is shown by the sum of a number of component potentials, anywhere within the soil-plant system

$$
\psi=\psi_{\pi}+\psi_{P}+\psi_{g}+\left[\psi_{\tau}\right]
$$

where $\psi_{\pi}$ is the osmotic potential, $\psi_{P}$ is pressure potential, $\psi_{g}$ is the gravitational potential, and $\psi_{\tau}$ is the matric potential. Practically, measurements of plant water potential are primarily conducted by means of either psychrometric methods or by means of the pressure chamber. Scholander et al. (1965) developed a measuring method to detect pressure gradient in the xylem part of plants, which became a robust and widely accepted reference technique, with a $0.1 \mathrm{MPa}$ estimated measurement accuracy (Waring and Cleary, 1967). It also has disadvantages being slow and labour intensive (therefore expensive, especially for predawn measurements) as well as unsuitable for automation (Jones, 2004). It is often claimed, that leaf water potential $\left(\psi_{\text {leaf }}\right)$ is a rig- 
orous and generally applicable measure of plant water status (Jones, 1990a), however he noted that rapid temporal fluctuations are often observed as a function of environmental conditions (such as passing clouds). As various authors propose, pressure chamber measurement of xylem water potential or stem water potential can offer a partial solution to the variability of leaf water status, however diurnal short-term fluctuations in $\psi_{\text {leaf }}$ are often greater than long term $\psi_{\text {leaf }}$ differences. In this article $\psi_{\text {leaf }}$ is referred to as xylem water potential of a leaf, measured by a pressure chamber. Indirect water status measurements contain physiological responses such as photosynthetic rate reduction detections. Such methods are often valuable in agronomic practice because it is often possible to derive repeatable calibration curves to allow estimation of water status for a given cultivar. Using the plant responses rather than direct measurements of soil water status gives a greater precision in the stress sensing (Jones, 1990b). A general limitation to plant-based methods is that those usually do not give information on 'how much' treatment (e.g. irrigation) to apply at a time, only whether or not treatment is needed. The use of a plant-based indicator needs the definition of a reference value, beyond which treatment is necessary. Such reference values are commonly determined for plants growing under non-limiting soil water supply (Fujino et al., 2002). In the recent study such indicators were used as control plants. As studies like Cohen et al. (2005) show, leaf water potential can be well estimated by combining temperature values derived from thermal images with spatial analysis, although in terms of expense and availability thermal cameras are uncommon in everyday agricultural practice. Pigment absorption definitely determines leaf spectral properties at visible range. Photosynthetic pigments absorb, transmit and reflect electromagnetic irradiation, thus define leaf optical character that enables studying a plant's physiological and photosynthetic properties by remote sensing (Field et al., 1994). The increasingly precise spectral evaluation of the vegetation at both leaf and field scale enables the detection of drought stress caused by nitrogen and water deficiency (Peñuelas et al., 1994). The term 'field spectroscopy' was introduced by Longshaw (1974) and had been accepted widely among the authors. In the frame of field spectroscopy the interrelationships between a plant's spectral characteristics and physiological attributes can be studied. For spectral calibration, the usage of a reflectance standard with known specral characteristics is necessary in order to be able to standardize canopy pixel intensity and to compare with other data sets. Many materials were proposed as reflectance standards (Milton, 1987), Gray Cards (GC) (Eastman Kodak, 1983) are often used in this role, especially in cost-conscious applications (Milton, 1989). Apart from GC advantages of low cost and easy availability, its spectral characteristics are well known, and its absolute reflectance is similar to that of vegetation. In the case of field-scale imagery, the calibrational use of a concrete block surface can be considered reasonable, Anderson and Milton (2005) found its reflectance factor response predictable. Regarding the correlation between leaf reflectance and water stress, when studying cucumber leaf spectral re- 
sponse on drought stress, Fereres and Goldhamer (2003) found a very slight reflectance change caused by a mild $(-1.2 \mathrm{MPa})$ water stress with reversible damage. There were a significant reflectance response at severe (-7.1 MPa) stress, however damage became irreversible much sooner, at -1.5 MPa level. Investigating drought stress effects on satsuma mandarin fruit, Yakushiji et al. (1998) showed that moderate stress resulted the highest sugar accumulation compared to severe stress and well-watered plants. In the case of peach, Besset et al. (2001) noted that true effects of major water stress on peach trees has still not been correctly evaluated. However our experiences showed a favorable peach fruit colour development as an effect of water stress. In southwestern Japan - where plain land availability is seriously limited - mandarin is produced on hillsides where sunny microclimate, high annual precipitation (about $2300 \mathrm{~mm}$ ) and good soil drainage favors good quality. Mandarin is overproduced in Japan, in addition more than $90 \%$ of Japanese growers have an orchard smaller than 1 hectare in size. Faced with this, high product quality is an important aspect and growers apply several techniques to improve it. Besides plastic/glasshouse covering and mounding, soil mulching is one of the most widespread agrotechnical treatment they use. Some extension officers express their doubts about the manufacturer of plastic DuPont Tyvek, who claim that two degrees Brix $\left({ }^{\circ} \mathrm{Bx}\right)$ sugar increase can be achieved by using it for mulching (Harty and Anderson, 1995). However our unpublished results show an approx. $2^{\circ} \mathrm{Bx}$ increase, and Iwagaki (1997), Morinaga et al. (2005) also report $1-2^{\circ} \mathrm{Bx}$ increase as well as an appropriate acid content and fruit rind colour improvement, just as well earlier and higher uniformity of colouring. Harty and Anderson (1995) claim that mulching can be successful only when using on shallow soils, its use on deep soils can even result poorer fruit quality. They also stated that laying down mulch earlier increases Brix but also increases acidity, which was also confirmed by our unpublished measurements. They suggest fruit storage to allow acidity to drop. Optimal quality mandarin has $12-14^{\circ} \mathrm{Bx}$ and $0.7-1.0 \%$ free acid content. $11-12^{\circ} \mathrm{Bx}$ fruits are still considered as good, however $>14^{\circ} \mathrm{Bx}$ sugar content is unnecessary. Rainfall conditions after the end of rainy season (end of July) seriously affects fruit quality, since that includes the sugar accumulation period which begins in the summer and continues up to harvest (Iwagaki, 1997). A significant part of the annual precipitation falls into this period. To prevent excessive soil moisture, and induce water stress, many southwestern Japanese mandarin growers cover soil with plastic mulch between rows in this period. For a more effective soil moisture, thus acid control, they install drip irrigation system under the plastic mulch optionally combine with liquid fertilizer system called 'drip irrigation and liquid fertilization method with year-round plastic mulching' (abbreviated DLYM, or 'Marudori' in Japanese) (Morinaga et al., 2005). The use of porous plastic mulch in Japanese mandarin orchards to dry soil is dated back to 1989, and in 1993 about 1700 hectares of satsuma mandarin orchard (3\% of total) were already mulched by some kind of plastic material (Iwagaki, 1997). Nonwoven polyethylene mulch perforated with submicron $(0.1-1.0 \mu \mathrm{m})$ holes 
allows soil moisture evaporation while drains rainwater. As a result, treated area's soil gradually dries out. In a DLYM system the application of precision irrigation results optimal sugar and acid content, high quality mandarin.

In the present study, the potential of using visible images for the in-field estimation of leaf water potential of mandarin and peach under different water stress conditions was investigated. The specific aims were (i) to investigate reflectance difference between water stressed and and control tree leaves (ii) to develop models for estimating leaf water potential based on visible images, thus supporting precision irrigation.

\title{
Materials and methods
}

\author{
Experimental layout and plant material
}

Measurements were made on satsuma mandarin, and peach. The field experiments were carried out at Wakayama Research Center of Agriculture, Forestry and Fisheries, Fruit Tree Experiment Station, Japan in the case of mandarin, and at Wakayama Research Center of Agriculture, Forestry and Fisheries, Fruit Tree Experiment Station, Persimmon and Peach Laboratory in the case of peach, during the summer and autumn of 2005. Times refer to Japan Standard Time.

Mandarin rootstock and variety were $c v$. Miyagawa Wase, that is an early variety, harvested between late October and late November. Trees were planted in 1976 on a south-east exposed hillside with a tree and row spacing $2 \mathrm{~m}$ and $4 \mathrm{~m}$ respectively. Tree heights were approximately $2.5 \mathrm{~m}$. In order to induce water stress, soil under mulched trees and between those rows was covered by Tyvek plastic cover (manufactured by DuPont Company) from the end of July (Fig. 1), that is the end of the rainy season, until harvest, the end of November. Measurements were made on 28 and 29 September, 24 and 25 November, 2005.

Peach rootstock and variety were $c v$. Hakuho, were planted in 1993 with a control tree and row spacing $4.5 \mathrm{~m}$ and $5.5 \mathrm{~m}$ respectively. Mulched peach trees were grown in 40 liter pots in a greenhouse, control trees were grown under conventional conditions. Water stress was induced by suspending the watering of the mulched trees 2 days before the experiment. Measurements were made on 27 and 30 September, 2005. 


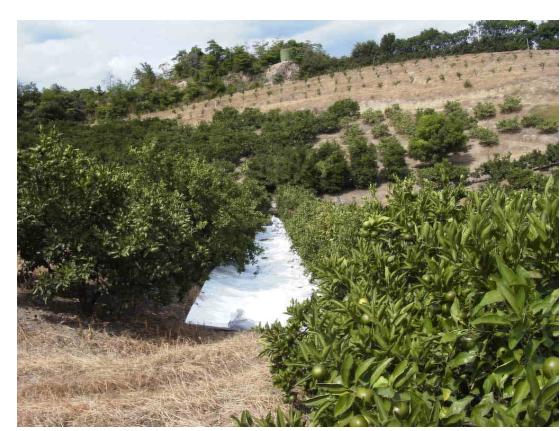

(a) Mulching between rows.

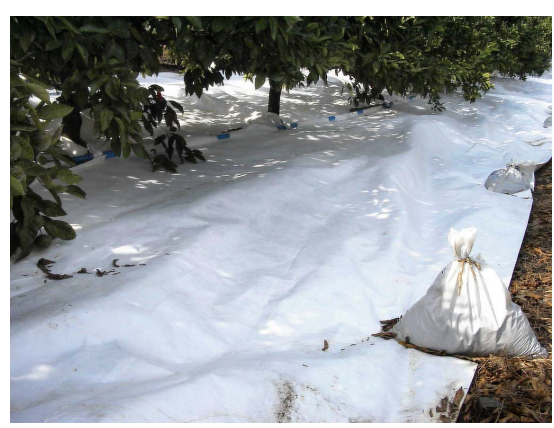

(b) Mulching between trees.

Fig. 1. Plastic mulch between mandarin rows in order to induce water stress.

Image acquisition and processing

Acquisition of multispectral images:

Multispectal images were taken with a three channel false colour CCD digital video camera (Silvacam model, manufactured by VTT Technical Research Centre of Finland), which was designed specially for vegetation remote sensing detection. The camera's detected channels were near infrared $\left(\rho_{N I R}=760-900 \mathrm{~nm}\right)$, red $\left(\rho_{R}=580-680 \mathrm{~nm}\right)$ and green $\left(\rho_{G}=490-580 \mathrm{~nm}\right)$ encoded respectively into standard RGB channels. It had a $720 \times 480$ pixel NTSC resolution and $29.97 \mathrm{fps}$ framerate. This resolution enabled to detect about $10^{4}$ pixel spatial information of each objects. Spectral calibration was necessary to enable the comparison of leaf reflectances recorded under different illumination conditions.

A GC $(20 \times 25 \mathrm{~cm})$ was placed into the camera's field of view. Instead of the nominal $18 \%$ reflectance of the gray card, we calculated with the reflectances $23.73 \%, 19.66 \%$ and $19.25 \%$ in near infrared, red and green channels respectively, based on Milton (1989).

Figure 2a showes the experimental layout of leaf reflectance recording. A white paper was used as background to facilitate segmentation. The leaf does not have a totally diffuse surface. Under field irradiation conditions some parts of leaf area exhibited specular reflection as shown on Fig. 2b and 2c. We tried to avoid this effect, although we could not fully eliminate it.

Analyzing of multispectral images:

Raw digital multispectral image data was recorded in DV format on tape. Raw DV video files were captured into a computer for non-linear video editing and image processing. PC was equipped with a $3.00 \mathrm{GHz}$ Intel Pentium $4 \mathrm{CPU}, 1$ 


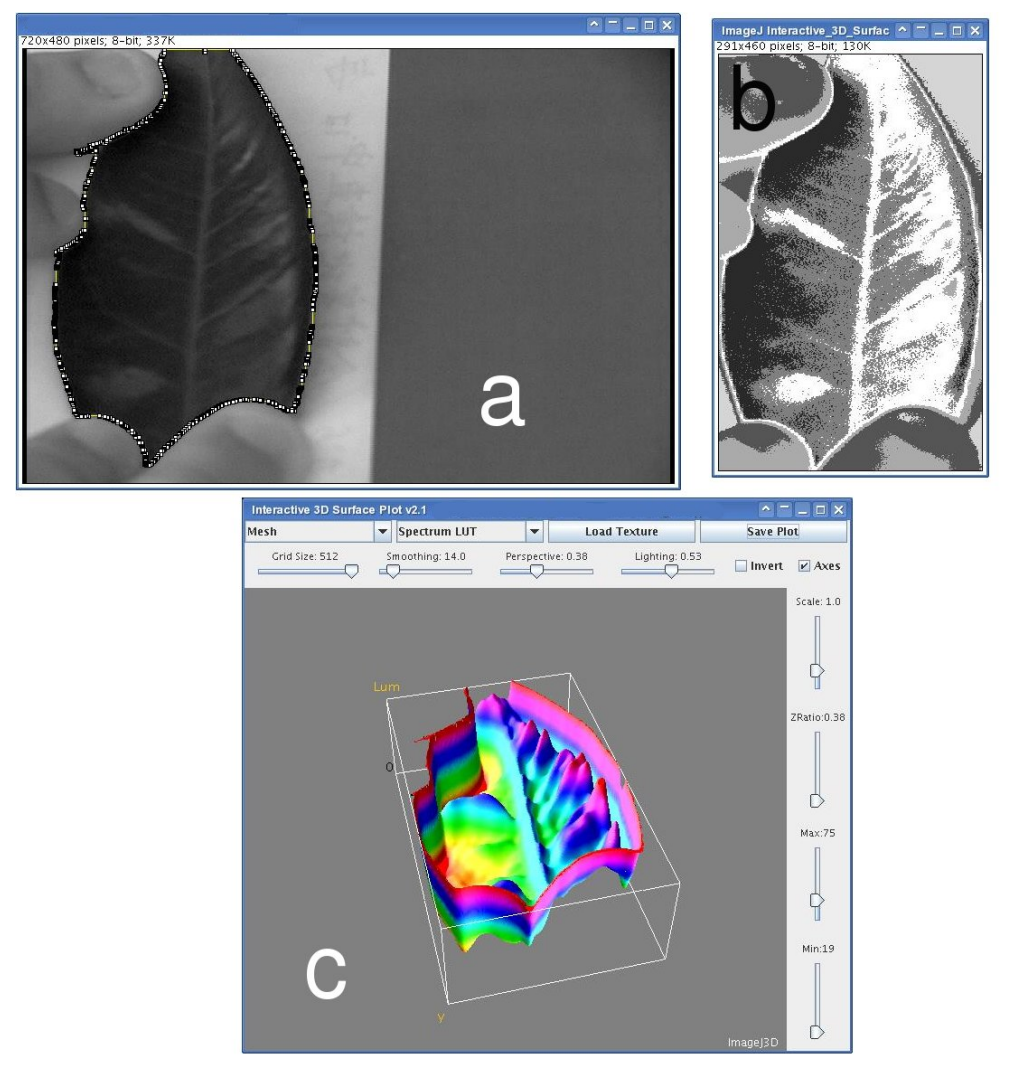

Fig. 2. Reflectance variation in a leaf image due to partially specular leaf surface characteristics. (a) Original image taken at red $\left(\rho_{R}=580-680 \mathrm{~nm}\right)$ channel showing reflectance measurement layout with segmented leaf at left and gray card at right. (b) 6-class k-means segmentation of the leaf object using k-means clustering algorithm. (c) Surface 3D plot of the leaf where luminance of pixels is interpreted as heights rendered by Interactive 3D SurfacePlot plugin (Barthel, 2007).

GB memory, running OpenSUSE Linux 10.1. Non-linear video authoring application Kino IEEE 1394 version 0.8.0 (Schirmacher et al., 2000) was used to capture digital video from miniDV tape played by Silvacam and saved as raw DV video file format. Kino was also used to export video frames as sequences of still images in lossless PNG file format. Each image sequence contained $\mathrm{n}=300$ still images. The number of images processed simultaneously was limited only by the memory of the computer. For analyzing the sequences of still images (hereafter: stack) ImageJ version $1.37 \mathrm{~d}$, a Java-based image processing software was used (Rasband, 1997). In order to achieve stable operation, a $694 \mathrm{MB}$ memory usage limit was specified for ImageJ, that enabled loading stacks of 300 images per leaf into the memory. Processing a stack completely loaded into memory ensured high-speed analysis. Image analysis was fast. During image analysis $10^{7}$ of the order of pixel per second analysis speed was reached, thus only a few seconds were needed to process a stack. Pixel intensity is the spatial unit that represents reflectance in remote sensing. In the course of segmentation as part of the image analysis process, pixels were classified into a foreground group, that contained useful information (leaf and 


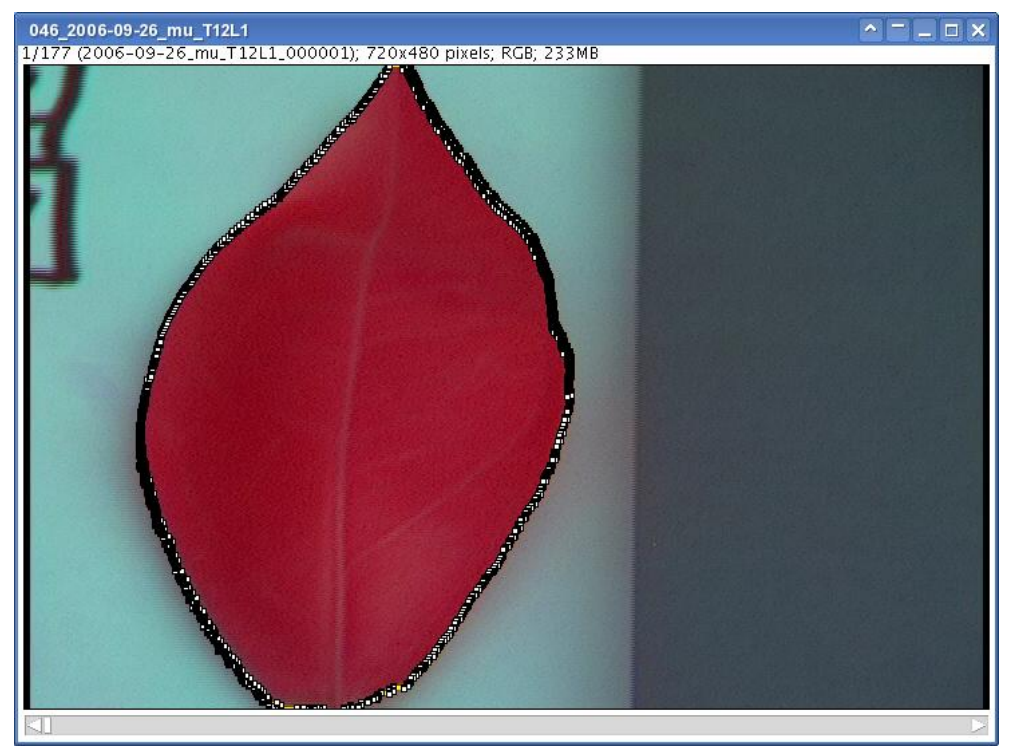

Fig. 3. Calculated exact outline of a false colour image leaf object using SegmentingAssistant plugin.

GC), and a background group, that did not. In order to select the maximum number of foreground pixels, a segmentation algorithm was used to detect the outline of an object in an image. Leaf and GC objects were segmented out of each image by using a modified SegmentingAssistant plugin (Miller, 2002) written for ImageJ. Due to the large amount of still images, the highest possible level of image segmenting automation was necessary. Requiring only four input parameters per stack, the algorithm effectively performed the automated segmentation task (Fig. 3), yielding the mean pixel intensities of an object in all three channels.

Field-scale imagery required a different segmentation technique, which also had to be automated as much as possible. In this case, stacks were segmented by K-means Clustering algorithm (MacQueen, 1967).

\section{Plant physiological measurements}

In order to detect plant water status, the widely accepted reference pressure chamber-based leaf water potential measurement technique (Jones, 2004) was used. Leaf water potential measurements were conducted with a pressure chamber (Model 600, PMS Instrument Company), following the same procedure as outlined by Cleary et al. (2006) . The timeconsuming pressure chamber measurement set the limit of the daily leaf sample to about $n=50$. Generally there were $n=24$ replications per treatment. On days 27 and 28 September, there were four replications per tree. Since no significant $\psi_{\text {leaf }}$ variability was found inside a tree, two leaves per tree - one from the sunny and one from the 
shaded side of canopy - were collected after 27 September. Leaves from sunny and shaded side of canopy did not show significant $\psi_{\text {leaf }}$ difference, as well as leaves that were sampled from different canopy heights. Spring leaves were selected and individually placed into plastic wraps, accompanied with wet tissues in order to avoid drying. Both were placed into an outer plastic bag. As Jones (2007) points out, the absolute value of $\psi_{\text {leaf }}$ varies diurnally over perhaps an order of magnitude, and we found that variation different in case of control and mulched trees. We measured equal predawn control and mulched $\psi_{\text {leaf }}$ values. During the morning both control and mulched $\psi_{\text {leaf }}$ increased, but the extent of control increased more. At midday mulched tree reached its daily $\psi_{\text {leaf }}$ maximum, as well as the difference between control and mulched $\psi_{\text {leaf }}$ was in maximum. During the afternoon, the control $\psi_{\text {leaf }}$ continued to increase, at about $15 \mathrm{~h}$ it approached the mulched's, their difference decreased. From $17 \mathrm{~h}$ control tree started to regenerate, its $\psi_{\text {leaf }}$ was restored at midnight. Mulched tree's $\psi_{\text {leaf }}$ restored slowlier than control's. Two components constitute $\psi_{\text {leaf }}$ : a short term daily component, and a long term component, related to mulching. To measure long term component, afternoon sampling seemed to be the most suitable time, then $\psi_{\text {leaf }}$ difference was caused mainly by the long term $\psi_{\text {leaf }}$ component.

\section{Results and discussion}

Differences between control and stressed leaves

Control and mulched leaves' $\psi_{\text {leaf }}, \rho_{R}$ and $\rho_{G}$ values were evaluated by significance test in order to evaluate their differences. Mean differences and the results of significance testing are summarized in Table1. Stress treatment effects on $\psi_{\text {leaf }}, \rho_{R}$ and $\rho_{G}$ were statistically significant on all measurements except $\rho_{G}$ on 29 September (mandarin) and 30 September (peach). Treatments with larger differences in mean $\psi_{\text {leaf }}$ showed larger differences in $\rho_{R}$, $\rho_{G}$ mean leaf reflectance values, indicating the correlation between $\psi_{\text {leaf }}$ and red, green leaf reflectance. However, the more severe the water stress was, the more clearly it could be detected by sensing leaf reflectance at visible range. Consistently, no significant difference was found between the $\rho_{N I R}$ of control and mulched leaves.

Regression models, relationship between leaf reflectance and leaf water potential

The relationship between leaf reflectance and leaf water potential is shown in Fig. 4 for individual days. Higher correlations were found between $\rho_{R}, \rho_{G}$ and 
Table 1

Mean difference of control and mulched leaf water potential and $\rho_{R}, \rho_{G}$ leaf reflectance values. Values indicated with 'o' are significantly different at $\mathrm{P}=0.05$, values indicated with ' $\mathrm{x}$ ' are not.

$\begin{array}{lrrrr}\text { Treatment } & 27 \text { September } \mathrm{p} & 28 \text { September m } & 29 \text { September m } & 30 \text { September } \mathrm{p} \\ \psi_{\text {leaf }}[\mathrm{MPa}] & -1.96 \mathrm{o} & -0.35 \mathrm{o} & -0.48 \mathrm{o} & -0.91 \mathrm{o} \\ \rho_{R}[\%] & 4.42 \mathrm{o} & 1.55 \mathrm{o} & 2.31 \mathrm{o} & 1.9 \mathrm{o} \\ \rho_{G}[\%] & 4.66 \mathrm{o} & 2.6 \mathrm{o} & 0.12 \mathrm{x} & 0.07 \mathrm{x} \\ \mathrm{n} & 24 & 48 & 48 & 48 \\ \text { Treatment } & 24 \text { November } \mathrm{m} & 25 \text { November } \mathrm{m} & & \\ \psi_{\text {leaf }}[\mathrm{MPa}] & -1.04 \mathrm{o} & -0.99 \mathrm{o} & \\ \rho_{R}[\%] & 4.02 \mathrm{o} & 1.52 \mathrm{o} & \\ \rho_{G}[\%] & 3.39 \mathrm{o} & 1.83 \mathrm{o} & & \\ \mathrm{n} & 48 & 48 & & \end{array}$

$\psi_{\text {leaf }}$ in the case of mandarin values measured on 24 November. Lower coefficient of determinations was observed on 27 September in the case of peach, and mandarin leaves measured on 28 September. Both slope and intercept of the $\rho-\psi_{\text {leaf }}$ relationship were found to have a stable tendency over time. The coefficient of determination may be improved by introducing other parameters into the model, such as canopy temperature or soil moisture. However, when considering a feasible application to use in horticultural production, then monitoring cost, automation possibility and complexity are important factors. Model accuracy level presented here can be adequate in the agricultural practice of precision irrigation, especially when coupling with a nearly real time detection. The usage of vegetation indices like Normalized Difference Vegetation Index (NDVI) or green NDVI was not proven reasonable. Shadows could be effectively segmented out by K-means clustering segmentation algorithm. We observed no correlation between $\psi_{\text {leaf }}$ and $\rho_{N I R}$, consequently $\rho_{R}$ and $\rho_{G}$ components were responsible for the correlation between $\psi_{\text {leaf }}$ and NDVI, green NDVI respectively.

\section{Field-scale imagery}

A false-colour composite image of the field (Fig. 5a) was used for segmentation in stack form. The number of clusters as a parameter for K-means clustering algorithm were determined interactively, the process result is shown on Fig. 5b. The segmentation objective was to keep only those pixels which represented sunlit parts of the canopy. The process above was followed by splitting the image into greyscale images NIR, red, green, encoded into R, G, B channels respectively. Recoding pixel intensities with false colours, mulched field 

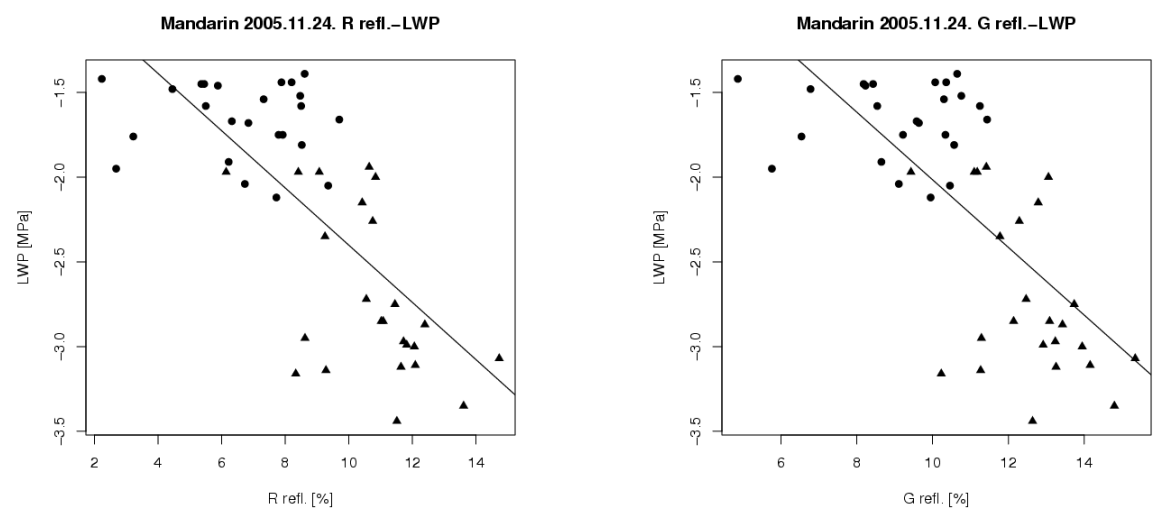

(a) $R^{2}=0.53 \quad y=-0.17 x-$ 0.71

(b) $R^{2}=0.51 \quad y=-0.2 x-$ 0.02
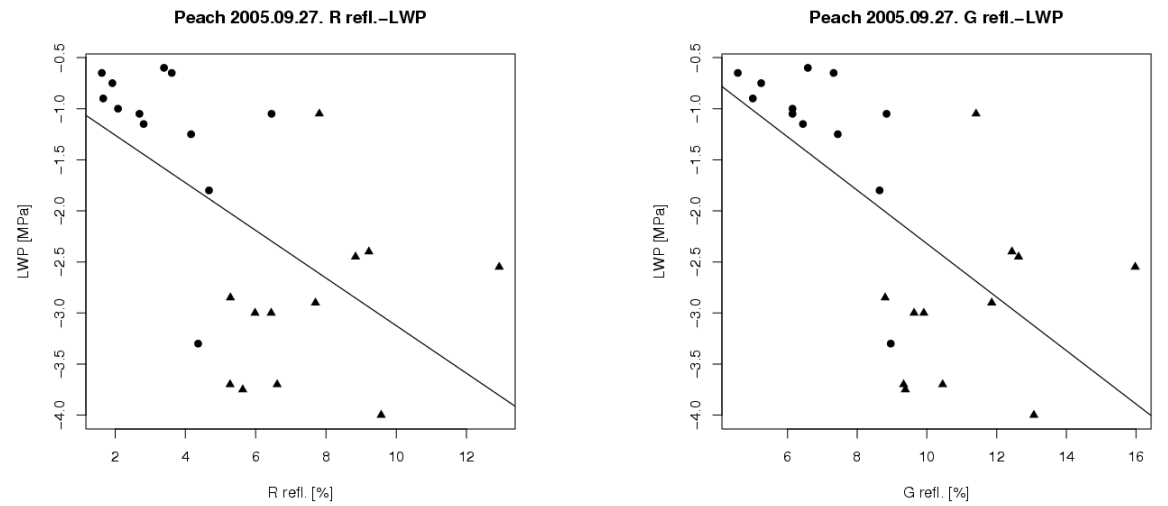

(c) $R^{2}=0.32 \quad y=-0.23 x-$ 0.79

(d) $R^{2}=0.41 \quad y=-0.26 x+$ 0.29
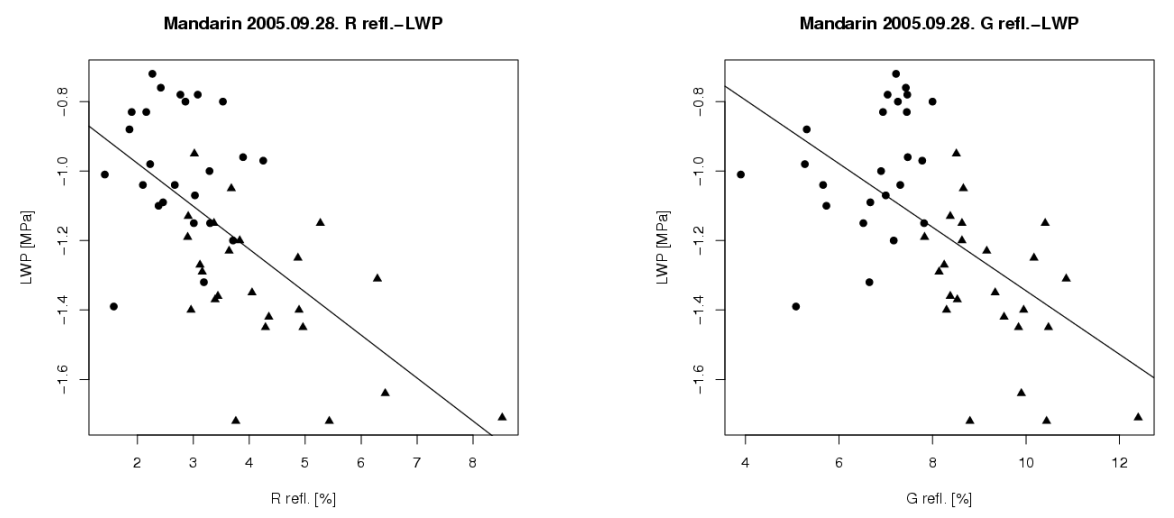
(e) $R^{2}=0.4$
$y=-0.12 x-$
0.73
(f) $R^{2}=0.35$
$y=-0.09 x-$
0.43

Fig. 4. Relationship between $\rho_{R}$ (R refl.), $\rho_{G}$ (G refl.) and $\psi_{\text {leaf }}$ (LWP) measured in individual plots on 24 Nov. (a) and (b), 27 Sep. (c) and (d), 28 Sep. (e) and (f). Control data are shown as $\bullet$, mulched data as $\boldsymbol{\Lambda}$. ANOVA: $n=48, R^{2}$ significant at $P<0.0001$ (a), $n=48, P<0.0001$ (b), $n=24, P<0.01$ (c), $n=24, P<0.001$ (d), $n=48, P<0.0001$ (e), $n=48, P<0.0001$ (f) 


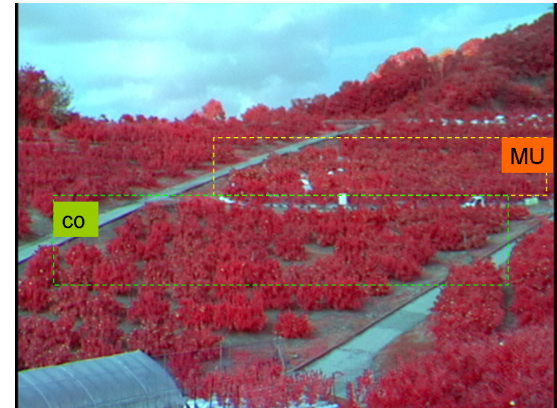

(a) Field scale multispectral image with control (CO) and mulched (MU) areas.

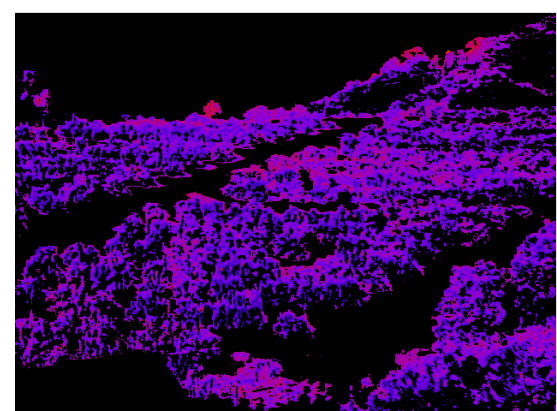

(c) Red channel of (b) with false colour. Lighter colour represents higher reflectance.

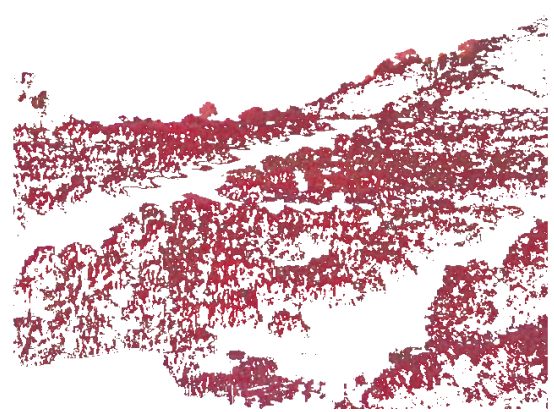

(b) K-means clustering segmentation result of image (a).

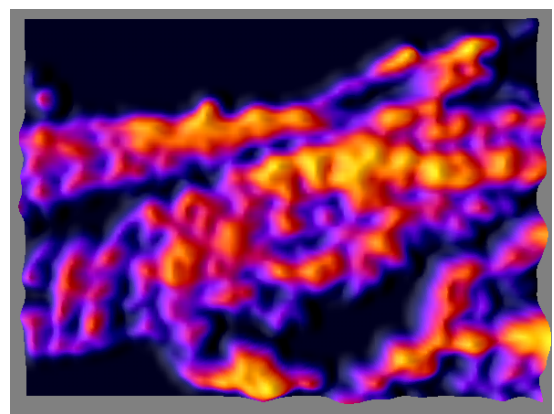

(d) Virtual 3D version of (b) Yellow $=$ high, blue = low reflectance.

Fig. 5. Field scale image recorded on 24 November representing reflectance difference between control and mulched orchard canopy areas.

area with higher canopy reflectance became visible (Fig. 5c). To emphasize the reflectance difference even more, a false colour 'topographic' style image transformation was applied in Fig. 5b resulting Fig. 5d where areas with virtually 'higher elevation' and lighter (yellow) colour indicate higher canopy reflectance at mulched area, while 'lower elevation' and darker (blue) colour mean lower canopy reflectance. These results can be obtained in real time or at least at frequent intervals, thus temporal and spatial monitoring and precision irrigation decisions can be made based on recent data. This is in contrast with other applications such as nitrogen fertilization by remote sensing, when a treatment decision is based on data measured in the previous year.

A concrete surface was used as reflectance standard in the case of field scale image recorded on 24 November (Fig. 5) in order to determine $\rho_{R}$ and $\rho_{G}$ of control and mulched area. Reflectance of the concrete surface was calibrated using a GC, and were found $30 \%, 26 \%$ and $24 \%$ in near infrared, red and green channels respectively. Using those standard reflectance values on the image shown on Fig. 5b, control and mulched areas' mean $\rho_{R}$ and $\rho_{G}$ values were calculated (Table 2 ). 
Table 2

Mean differences of control $(n=24)$ and mulched $(n=24)$ leaf water potentials as well as mean $\rho_{R}, \rho_{G}$ values.

$\begin{array}{lrrr}\text { Treatment } & 24 \text { November CO } & 24 \text { November MU } & \text { mean difference } \\ \psi_{\text {leaf }}[\mathrm{MPa}] & -1.67 & -2.71 & 1.04 \\ \rho_{R}[\%] & 8.97 & 9.71 & 0.74 \\ \rho_{G}[\%] & 10.32 & 10.67 & 0.35\end{array}$

\section{Conclusion}

This study demonstrated that (i) there was a reflectance difference between water stressed and non-water stressed mandarin and peach plants in red $\left(\rho_{R}=580-680 \mathrm{~nm}\right)$ and green $\left(\rho_{G}=490-580 \mathrm{~nm}\right)$ spectral channels both at individual leaf level and field-scale canopy level, that could be used as indicators of plant water stress; (ii) linear relationships existed also when $\psi_{\text {leaf }}$ was derived from image analysis of leaves in $\rho_{R}$ and $\rho_{G}$ channels.

Future studies should aim at (i) improving the water stress detection method presented in this paper to sense milder stress (ii) investigating how measuring accuracy varies under different environmental conditions, during a daily sampling period, and in relation with soil water difference, (iii) developing the $\rho_{R}-\psi_{\text {leaf }}$ and $\rho_{G}-\psi_{\text {leaf }}$ relationships into an automatized tool for precision irrigation management on a field-scale.

\section{References}

Anderson K; Milton E (2005). Characterization of the apparent reflectance of a concrete calibration surface over different time scales. Proceedings of the 9th International Symposium on Physical Measurements and Signatures in Remote Sensing (ISPMSRS). 17-19 October, Beijing, China, 41719

Barthel K U (2007). Interactive 3D SurfacePlot. Version 2.1 http://rsb.info.nih.gov/ij/plugins/surface-plot-3d.html Retrieved on: 2007.04.11.

Besset J; Genard M; Girard T; Serra V; Bussi C (2001). Effect of water stress applied during the final stage of rapid growth on peach trees (cv. Big-Top). Scientia Horticulturae, 91(3-4), 289-303

Cleary B; Zaerr J; Hamel J (2006): Guidelines for measuring plant moisture stress with a pressure chamber. (PMS Instrument Company). http://pmsinstrument.com/tutorials/Guidelines Booklet.pdf Retrieved on: 2007.05.08.

Cohen Y; Alchanatis V; Meron M; Saranga Y; Tsipris J (2005). Es- 
timation of leaf water potential by thermal imagery and spatial analysis. Journal of Experimental Botany, 56(417), 18431852

Eastman Kodak, (1983). Instructions For Use of the Kodak Neutral Test Card. Publication number 092-4-80-ABX (Rochester New York: Eastman Kodak Company)

Fereres E; Goldhamer D A (2003). Suitability of stem diameter variations and water potential as indicators for irrigation scheduling of almond trees. Journal of Horticultural Science and Biotechnology, 78, 139144

Field C B; Gamon J A; Peñuelas J (1994). Remote sensing of terrestrial photosynthesis. In: Schulze E D; Caldwell M M (Eds.): Ecophysiology of photosynthesis, Ecological Studies 100, 511-527

Fujino M; Endo R; Omasa K (2002). Nondestructive instrumentation of water-stressed cucumber leaves - comparison between changes in spectral reflectance, stomatal conductance and PSII yield and shape. Japanese Society of Agricultural Informatics, Agricultural Information Research, 11(2), $161-170$

Harty A; Anderson P (1995). Japanese Production Practices for Satsuma Mandarins. Orchardist of New Zealand 68(3), 40-43

http://www.hortnet.co.nz/publications/science/h/harty/jpsatsm.htm Retrieved on: 2007.05.08.

Iwagaki I (1997). Citrus production in Japan: new trends in technology. http://www.agnet.org/library/eb/440/eb440.pdf

Retrieved on: 2007.05.08.

Jones H G (1990a). Physiological aspects of the control of water status in horticultural crops. HortScience, 25, 1926

Jones H G (1990b). Plant water relations and implications for irrigation scheduling. Acta Horticulturae, 278, 6776

Jones H G (2004). Irrigation scheduling: advantages and pitfalls of plantbased methods. Journal of Experimental Botany, Water-Saving Agriculture Special Issue, 55(407), 2427-2436

Jones H G (2007). Monitoring plant and soil water status: established and novel methods revisited and their relevance to studies of drought tolerance. Journal of Experimental Botany, 58(2), 119-130

Longshaw T G (1974). Field spectroscopy for multispectral remote sensing: an analytical approach. Applied Optics, 13, 1487-1493

MacQueen J B (1967). Some Methods for classification and Analysis of Multivariate Observations. Proceedings of 5-th Berkeley Symposium on Mathematical Statistics and Probability, Berkeley, University of California Press, 1, 281-297

Miller M A (2002). SegmentingAssistant http://php.iupui.edu/ mmiller3/ImageJ/SegmentingAssistant.html Retrieved on: 2007.04.10.

Milton E J (1987). Principles of field spectroscopy. International Journal of Remote Sensing, 8(12), 1807-1827

Milton E J (1989). On the suitability of Kodak neutral test cards as re- 
flectance standards. International Journal of Remote Sensing, 10(6), 10411047

Morinaga K; Sumikawa O; Kawamoto O; Yoshikawa H; Nakao S; Shimazaki M; Kusaba Sh; Hoshi N (2005). New Technologies and Systems for High Quality Citrus Fruit Production, Labor-saving and Orchard Construction in Mountain Areas of Japan. Journal of Mountain Science, 2(1), 59-67

http://www.unu.edu/env/plec/marginal/proceedings/MorinagaCH15.pdf Retrieved on: 2006.12.18.

Peñuelas J; Gamon J A; Fredeen A L; Merino J; Field C B (1994). Reflectance indices associated with physiological changes in nitrogen- and water-limited sunflower leaves. Remote Sensing of Environment, 48, 135-146

Rasband W S (1997-2006): ImageJ. U. S. National Institutes of Health, Bethesda, Maryland, USA, http://rsb.info.nih.gov/ij/

Schirmacher A; Dennedy D; Yates Ch; Dydensborg M D (2000-2005): Kino. http://www.kinodv.org Retrieved on: 2007.04.10.

Scholander P F; Hammel H T; Bradstreet E D; Hemmingsen E A (1965). Sap pressure in vascular plants. Science, 148, 339-346

Slatyer R O; Taylor S A (1960). Terminology in plant- and soil-water relations. Nature, 187, 922-924

Waring R H; Cleary B D (1967). Plant moisture stress: evaluation by pressure bomb. Science, 155, 1248-1254

Yakushiji H; Morinaga K; Nonami H (1998). Sugar Accumulation and partitioning in Satsuma mandarin tree tissues and fruit in response to drought stress. Journal of the American Society for Horticultural Science, 123(4), 719-726 